\title{
Fall Detection for Elder People Using Single Inertial Sensor
}

\author{
Wei Zhuang ${ }^{1, a}$, Xiang Sun ${ }^{1, b}$ and Dong Dai ${ }^{2, c}$ \\ ${ }^{1}$ Nanjing University of Information Science \& Technology, China \\ ${ }^{2}$ Southeast University, China \\ azw@nuist.edu.cn, b18362097621@163.com, 'daidong2069@qq.com
}

\begin{abstract}
Keywords: Fall Detection; Behavior Recognition; SVM; Inertial Sensors
\end{abstract}
\begin{abstract}
This paper presents a fall detection system for elder people using wearable single inertial sensor. In contrast with multiple wearable sensors, we deploy only one 3-axis accelerator on human body for continuously monitoring fall incident. Support Vector Machine is exploited to be the classifier for predicting the behavior. The dedicated features including intensity of acceleration, ascending coefficient and descending coefficient are selected for training model. The experimental results have shown that the positive detection rate can reach $92.5 \%$ after optimizing SVM parameters.
\end{abstract}

\section{Introduction}

Falling is the fourth cause of death in China, while ranking first for people older than 65. It is reported that more than $30 \%$ of people over the age of 65 fall down at least once a year. As they getting older, the probability of falling will gradually increase, and the annual incidence of the elder people over the age of 80 reaches as high as $50 \%$, while $5 \%-10 \%$ falls can lead to fractures. With the increase of age, the disability ratio and mortality rise sharply. Therefore, reliable fall prevention and accurate detection is one of most important technologies. It can precisely determine falling behavior, evaluate the severity of the fall, and immediately inform medical personnel or family members. At present, the technology about fall behavior analysis and recognition can mainly divide into two categories: one is fall behavior recognition method based on visual information; the second is motion analysis and recognition based on wearable inertial sensors.

With the development of efficient image processing technology, visual information based behavior monitoring system has been widely concerned. Through one or more cameras collecting continuous images of the target, it can extract particular features of those images so as to estimate the behavior characteristics. So far, most of the algorithms based on visual information use raw video data, while some researchers use infrared or contour detecting camera to protect privacy information of the target. Rougier et al. designed proper thresholds by tracking the change of the head shape to detect fall behavior [1]. Yu et al. obtained body falling images with background subtraction method. Using support vector machine (SVM) for different types of histogram, this approach can increase the positive detection rate to $97.08 \%$ [2].

However, this technique is usually limited by surrounding environments and equipment. For instance, the image acquisition devices need to be deployed on or around the target, which is difficult to be realized in outdoor behavior monitoring system, In addition, visual sensors have large power consumption while wearable nodes are often supplied through micro batteries. Generally, visual based fall detection system increases the cost of the platform to achieve the goal.

In recent years, with the rapid development of micro-electro-mechanical sensor technology, using micro inertial device to detect human behavior has gradually become possible. Fall monitoring system based on inertial devices are mostly using tiny accelerometer, gyroscope and tilt sensor for acquiring posture information of the target, and using proper thresholds for detecting fall occurrence. For example, Purwar et al. exploits a three-axis accelerometer to obtain the body's angle with the ground, then determine if fall occurs or not [3]. Compared to the visual collection device, MEMS devices relatively have low energy consumption, smaller size, therefore more suitable for behavior detection of outdoor environment. 
However, those methods are usually based on multiple nodes system, that is, the target needs to wear more than two inertial sensor nodes on the body. For instance, Atallah et al. develop a fall monitoring platform with wearable nodes deployed in five locations including the chest, waist, wrist, leg, and foot [4]. Although it is concluded that the positive detection rate is as high as $99.8 \%$, the testers are often only willing to wear single node in the real daily life. The deployment location and related feature selection of single node detection system have more requirement than multiple nodes system, because it is harder to distinguish the fall incidence from similar behavior such as couching and bowing. Tong et al. present posture analysis by deploying a three-axis accelerometer node on the chest while they use Hidden Markov Model to identify the fall incident [5]. However, their study does not address the experimental results for other parts of the body, and the chosen features of HMM classifier is only the intensity of the acceleration.

In our work, we exploit Supporting Vector Machine (SVM) as the fall behavior classifier based on single inertial sensor. The contribution of our works mainly focus on presenting an effective system to determine fall incidents with low-power consumption and light-weight computation. Inspired by aforementioned methods, the three-axis acceleration sensors are deployed in each part of the body for evaluating the optimal location, and three features are adopted to add into SVM classifier for recognition.

\section{System Structure}

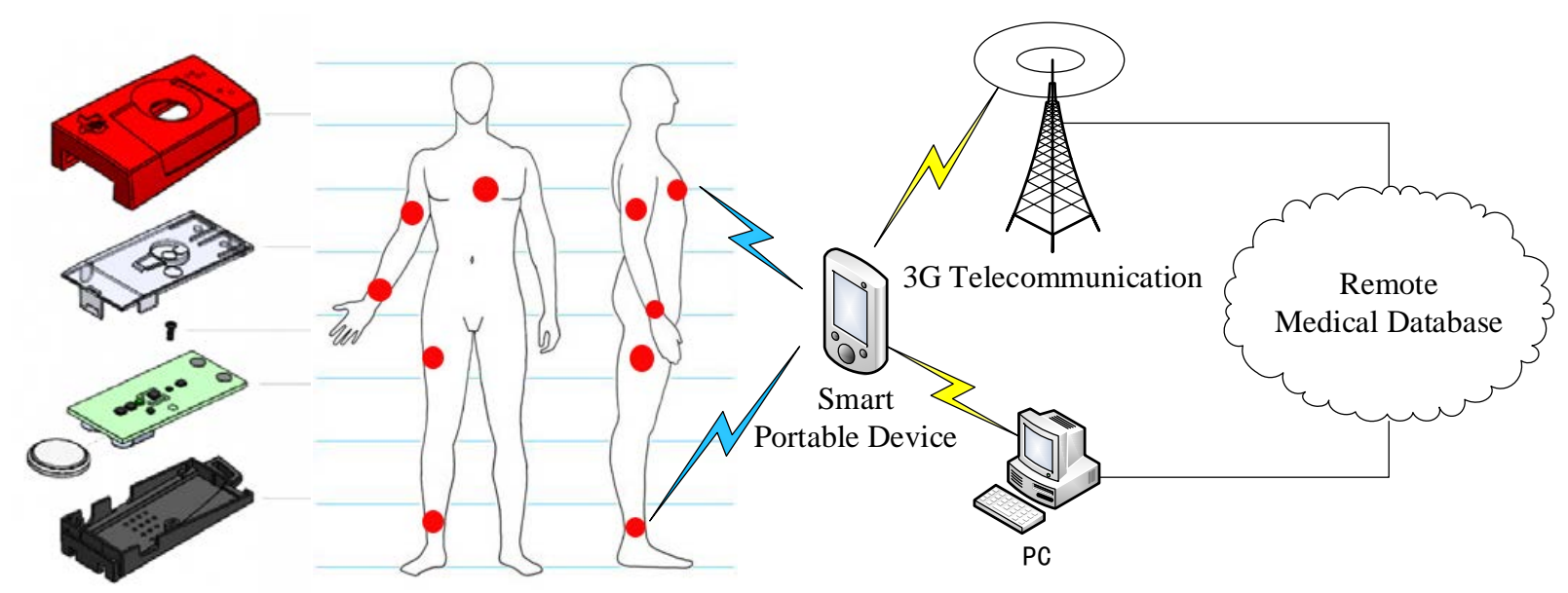

Fig 1. The diagram of fall detection system for elder people

Fall Detection System. A fall incident detection and recognition system is proposed in this paper as shown in Fig.1. Whether indoor or outdoor, the system can accurately acquire vital motion signs and immediately send out warning message to portable devices (smart phone, PDA, intelligent glass etc.). Then, these messages will be broadcasted by 3G telecommunication network or pc sever, pushed into remote medical database for noticing medical personnel.

Hardware and Software. The wearable inertial sensors are shown in left of Fig.1, which is produced by TI, named SensorTag. The node is powered by one CR2032 battery with low energy consumption ability and sufficient computing for driving sensors. This type of node can be mounted on human body, while the deployment location does matter. We assume five potential locations as the raw data sources including chest, upper arm, wrist, thigh, and ankle. The selected testing results are presented in sections later.

The system framework is presented in Fig.2. After system initiating (sensor calibration or startup), it will train the SVM model for individual tester, then construct fall database with a fixed format. The accelerators are keeping measuring the 3-xias inertial signals with a slide window during all the time. The system can extract the selected features and send into SVM prediction module. If it is beyond the determined threshold, the system could send out a warning message to mobile networks. 


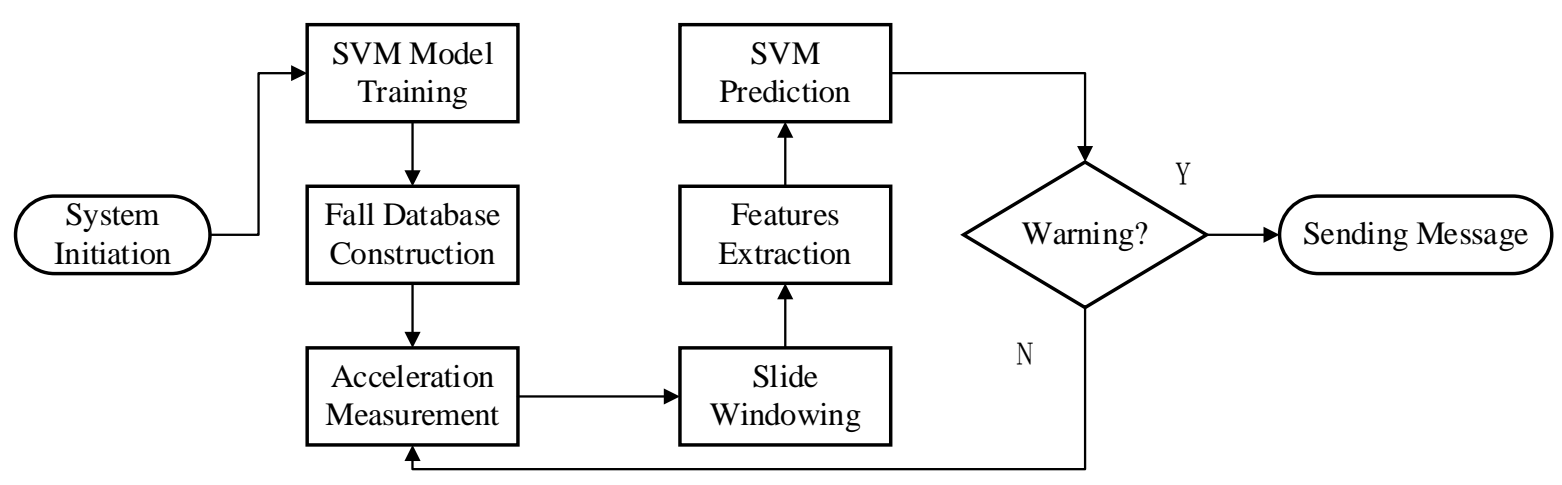

Fig 2. The software framework of fall detection system

\section{Features Selection}
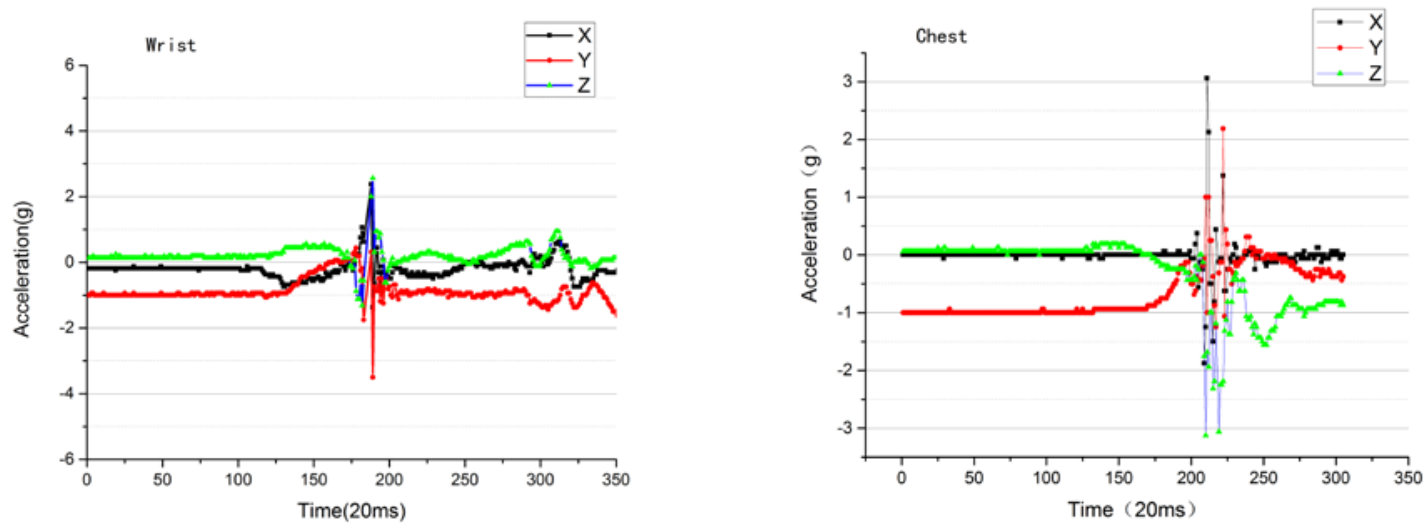

Fig.3 Different intensities from chest and wrist

Intensity of Acceleration. Considering the low-energy consumption, we assign the first feature as intensity of three acceleration values. These data can be directly acquired through wearable inertial sensor. The intensity is always a benchmark for evaluating motion indication that is presented as:

$$
\text { Int }=\sqrt{a_{x}^{2}+a_{y}^{2}+a_{z}^{2}}
$$

Fig. 3 shows the difference between three axis acceleration values from wrist and chest. We compare all data in five position (Fig.1 shows the location), then conclude that the intensity from chest is the most representing indication of all. Those data are chosen in tanning and prediction module.

In order to distinguish high intense motions from fall behavior such as fast running, up-and-down exercise or turning. We also add ascending coefficient into selected feature set. This feature represents the nearest slope of rising from bottom to top before the wave peak. It describes the sudden occurrence of fall incident. Likewise, the decaying of intensity is also distinct with other motions. We add descending coefficient into the feature set, meaning the nearest slope of dropping from top to bottom after the wave peak. These features with low computing complex have shown its suitability for SVM training and prediction.

\section{Experiments}

Eight students (4 male, 4 female) have volunteered for testing our fall incident detection system. To simulate the real daily life as much as possible, testers are demanded to walk in a corridor and suddenly fall down into a gym mattress. We set up three scenarios such as stripped by obstacles, pushed by other people and fainting. Three TI SensorTag sensors are mounted on each testers' chest, wrist and thigh while the real-time acceleration packets are saved in a smart phone through BLE 
network. The dedicated SVM classifier can simultaneously format these data and send out a result using our training model. If there is a positive result, it will send out a warning message into screen.

Table 1 and Table 2 are the training dataset of samples for SVM classifier using our selected features, respectively. As a result, the accuracy of determined fall is $85.1 \%$. After the parameter $C$ and gamma of SVM classifier being optimized, that is, $C=256$, gamma $=0.005$, the positive rate impressively rises up to $92.5 \%$.

Table 1. Samples for determined fall

\begin{tabular}{ccc} 
Intensity & Asc Coeff & Desc Coeff \\
\hline \hline 4.0821639 & 1.029245263 & -0.08284102 \\
11.585619 & 1.135540664 & -2.050054535 \\
3.7629983 & 0.04452821 & -0.414724497 \\
3.2745467 & 0.063690876 & -0.817096318 \\
4.1240529 & 0.108281224 & -0.87705912 \\
7.6034532 & 3.18403578 & -3.447320646 \\
7.0660942 & 0.055218925 & -0.576619567 \\
8.4562292 & 0.154698168 & -1.755016636 \\
10.617093 & 0.150203404 & -2.561135999
\end{tabular}

Table 2. Samples for non-determined fall

\begin{tabular}{ccc} 
Intensity & Asc Coeff & Desc Coeff \\
\hline \hline 3.54325945 & 0.553041017 & -0.013924779 \\
3.26259578 & 0.006936865 & -0.00122 \\
4.65446694 & 0.02209198 & -0.00692676 \\
3.76818507 & 0.00718884 & -0.000639708 \\
4.16880154 & 0.000601779 & -0.023231037 \\
1.25778824 & 0.0000301 & -0.000389649 \\
1.20869248 & 0.000461241 & -0.003413 \\
1.27015009 & 0.004621544 & 0.033157536 \\
1.27628612 & 0.122149534 & -0.072451098
\end{tabular}

\section{Conclusion}

The intension of our work is to testify the fall detection system using only one wearable sensor. The contribution of our works mainly focus on presenting an effective system to determine fall incidents with low-power consumption and light-weight computation. Three-axis accelerators are deployed on the body for evaluating the optimal location, and three features are adopted to add into SVM classifier for recognition. From the experimental results, we can conclude that a satisfied positive rate can be achieved via selecting proper features. The future works will focus on designing novel nodes attached on body with small size and long-term standby abilities.

\section{Acknowledgement}

This paper is supported by the Natural Science Foundation of the Jiangsu Higher Education Institutions of China (Grant No. 12KJB510010) , Research on Key Technologies of Remote Health Monitoring based on Body Sensor Networks (Grant No. 2014H040), Nanjing University of Information Science and Technology Research Foundation (Grant No. 2012X032).

\section{References}

[1] Rougier C, Meunier J, St-Arnaud A, et al. Monocular 3D head tracking to detect falls of elderly people[C]. 28th Annual International Conference of the IEEE, 2006: 6384-6387.

[2] Yu M, Rhuma A, Naqvi S M, et al. A posture recognition-based fall detection system for monitoring an elderly person in a smart home environment [J]. Information Technology in Biomedicine, IEEE Transactions on, 2012, 16(6): 1274-1286.

[3] Purwar A, Myllyla R, Chung W Y. A wireless sensor network compatible triaxial accelerometer: Application for detection of falls in the elderly [J]. Sensor Letters, 2008, 6(2): 319-325.

[4] Tolkiehn M, Atallah L, Lo B, et al. Direction sensitive fall detection using a triaxial accelerometer and a barometric pressure sensor[C]. 2011 Annual International Conference of the IEEE, 2011: 369-372.

[5] Tong L, Song Q, Ge Y, et al. HMM-based human fall detection and prediction method using tri-axial accelerometer [J]. Sensors Journal, IEEE, 2013, 13(5): 1849-1856. 